\title{
Reliability Modeling and Evaluation of Electric Vehicle Motor by Using Fault Tree and Extended Stochastic Petri Nets
}

\author{
Bing Wang, ${ }^{1,2}$ Guangdong Tian, ${ }^{2}$ Yanping Liang, ${ }^{1}$ and Tiangang Qiang ${ }^{2}$ \\ ${ }^{1}$ School of Electrical and Electronic Engineering, Harbin University of Science and Technology, Harbin, Heilongjiang 150080, China \\ ${ }^{2}$ Transportation College, Northeast Forestry University, Harbin, Heilongjiang 150040, China \\ Correspondence should be addressed to Guangdong Tian; tiangd2013@gmail.com
}

Received 20 March 2014; Accepted 6 April 2014; Published 30 April 2014

Academic Editor: Weichao Sun

Copyright (c) 2014 Bing Wang et al. This is an open access article distributed under the Creative Commons Attribution License, which permits unrestricted use, distribution, and reproduction in any medium, provided the original work is properly cited.

Performing reliability analysis of electric vehicle motor has an important impact on its safety. To do so, this paper proposes its reliability modeling and evaluation issues of electric vehicle motor by using fault tree (FT) and extended stochastic Petri nets (ESPN). Based on the concepts of FT and ESPN, an FT based ESPN model for reliability analysis is obtained. In addition, the reliability calculation method is introduced and this work designs a hybrid intelligent algorithm integrating stochastic simulation and NN, namely, NN based simulation algorithm, to solve it. Finally, taking an electric vehicle motor as an example, its reliability modeling and evaluation issues are analyzed. The results illustrate the proposed models and the effectiveness of proposed algorithms. Moreover, the results reported in this work could be useful for the designers of electric vehicle motor, particularly, in the process of redesigning the electric vehicle motor and scheduling its reliability growth plan.

\section{Introduction}

Along with increasing problems of energy and environment, more and more countries develop related policies to handle these changing and disturbing issues, that is, developing newenergy and low-carbon vehicles, implementing the remanufacturing, reuse, and recycling of waste products, and performing green transportation technologies [1-5]. Electric vehicle, as an important and green transportation tool, has been widely attracted by more and more researchers [6]. Motor is one of the key components of electric vehicle. Its reliability has an important impact on the system safety. Designers have well-recognized the importance of electric vehicle reliability, but to our best knowledge a detailed reliability analysis is still missing. Although the faults have been reduced in the last few years by some measures, the faults still affects the safety of vehicles, and faults of mechanical system occupy a large proportion of all the faults.

In the present literature, most of the current researches have discussed electric and electronic system issue and reliability prediction analysis of the electric vehicle. For example, P. Liu and H. P. Liu present a permanent-magnet synchronous motor drive system of electric vehicles [7]. Peng et al. discuss driving and control problems of torque for direct-wheel-driven electric vehicle with motors in serial [8]. Quinn et al. present the effect of communication architecture on reliability [9]. Zhu et al. present a grey prediction model of motor reliability of electric vehicle [10]. They propose a grey prediction model of electric vehicle motor based on particle swarm optimization [11]. In addition, Zhu et al. discuss the reliability modeling method of solar array based on the fault tree (FT) analysis method [11].

It can be seen from the above literatures that the current research on reliability modeling of electric vehicle motor is limited to FT analysis method. There is no doubt that FT analysis has been widely employed as a powerful technique to evaluate the safety and reliability of complex systems by many scholars $[12,13]$. However, FT analysis has some limitations in reliability analysis. Firstly, in FT analysis, the probabilities of basic events must be known before analysis. Thus based on this assumption, the reliability analysis of the system is only a probability decision-making process 
and cannot achieve the real-time description of reliability information $[14,15]$. Secondly, it is not easy for FT analysis to conduct further quantitative analysis automatically due to the lack of effective means of mathematical expression. Thirdly, FT analysis cannot find the dynamic description of fault information of the system precisely and cannot describe the propagation process of fault information.

The Petri net is one of the mathematical modeling approaches for the description of distributed systems, which consists of places, transitions, and directed arcs [16, 17]. Many extensions to the Petri nets have been successfully developed and applied in analyzing fault diagnosis, automated manufacturing systems, and product disassembly [18-21]. The extended stochastic Petri net is a high level one; it has been used to establish models of reconfigurable manufacturing system and network attack due to its better information expression ability and dynamic description performance of process features $[22,23]$. Although some prior works [2224] have proposed to use extended stochastic Petri nets to solve reliability issues of nitric acid reactor feed and reconfigurable manufacturing systems, they merely analyze the average failure rate/life time of system; the real-time probability analysis/reliability issues of the system are not yet addressed by using this method. Moreover, for reliability issue of electric vehicle motor, we cannot find reference to handle this issue by the extended stochastic Petri nets method to the best knowledge of the authors. To do so, this work addresses reliability modeling and evaluation of electric vehicle motor by using extended stochastic Petri nets based on fault tree for the first time. Namely, the aim of this work is to find a new way to analyze reliability of electric vehicle motor.

The remainder of this paper is organized as follows: reliability model and establishment method of FT and extended stochastic Petri nets of the mechanical system are given in Section 2 . Section 3 presents the reliability analysis method and algorithm. In Section 4, taking an electric vehicle motor as an example, its reliability modeling and evaluation are presented. Section 5 concludes our work and describes some future research issues.

\section{FT Based Extended Stochastic Petri Nets (ESPN) Models for Reliability Analysis}

Reliability model is basis and premise for reliability analysis and evaluation; thus we first introduce the concept and establishment process of extended stochastic Petri nets model based on FT for reliability analysis. To easily establish it, the following method is proposed in this work. Namely, the FT model for reliability analysis is established based on its related concept, and then transformation rules of elements of FA to ESPN are defined. Finally, FT based ESPN model for reliability analysis is established.

2.1. FT Model for Reliability Analysis. FT is the most usual model of reliability analysis. Many references describe it in detail $[25,26]$. In this work, we only present its basic elements and schematic diagram.
2.1.1. Basic Elements of FT. Usually, FT is composed of a series of events and logic gates. The main events include the following.

Top event: it is the most undesirable system failure event and the object of the analysis. It is denoted by $\square$.

Middle event: it is the subsystem or component failure event and the cause of the top event. It is denoted by $\square$.

Basic event: it is the primary failure event and the cause of the top event or middle events. It is denoted by $\mathrm{O}$.

The main logic gates include the following.

Logic OR gate: it indicates that output event occurs if either one of the input events occurs. It is denoted by A.

Logic AND gate: it indicates that output event occurs only if all of the input events occur. It is denoted by

\section{Q}

There may be many other types of events and logic gates involved in complex system reliability analysis. However, for the sake of concisions, we only list the most commonly used ones here. For other types of events and logic gates, please refer to $[25,26]$.

2.1.2. FT Model of a Mechanical System. Based on the presented basic elements and logical relationship of fault occurrence, the schematic diagram of FT for reliability analysis of a mechanical system is presented next, as shown in Figure 1.

As shown in Figure 1, this FT is composed of 1 top event, 2 middle events, and 4 basic events. They are T, M1 and M2, and $\mathrm{B} 1, \mathrm{~B} 2, \mathrm{~B} 3$, and $\mathrm{B} 4$.

\subsection{ESPN Model for Reliability Analysis}

2.2.1. Concept of ESPN. The Petri net is a graphic modeling method, which is widely used in modeling and analyzing discrete event systems such as semiconductor manufacturing, transportation, and automated manufacturing systems. An ESPN is a high level one. It is a type of improved stochastic Petri nets with arbitrary distribution. Before giving the formal definition, we present the definition of PN introduced by Petri in 1962 [27-30].

A PN is a five-tuple $(P, T, I, O, m)$, where

$P=\left\{p_{1}, p_{2}, \ldots, p_{n}\right\}, n>0$, and is a finite set of places pictured by circles;

$T=\left\{t_{1}, t_{2}, \ldots, t_{s}\right\}, s>0$, and is a finite set of transitions pictured by bars, with $P \cup T \neq \Phi, P \cap T=\Phi$;

$I: P \times T \rightarrow N$ and is an input function that defines the set of directed arcs from $P$ to $T$, where $N=$ $\{0,1,2 \ldots\}$;

$O: T \times P \rightarrow N$ and is an output function that defines the set of directed $\operatorname{arcs}$ from $T$ to $P$; 


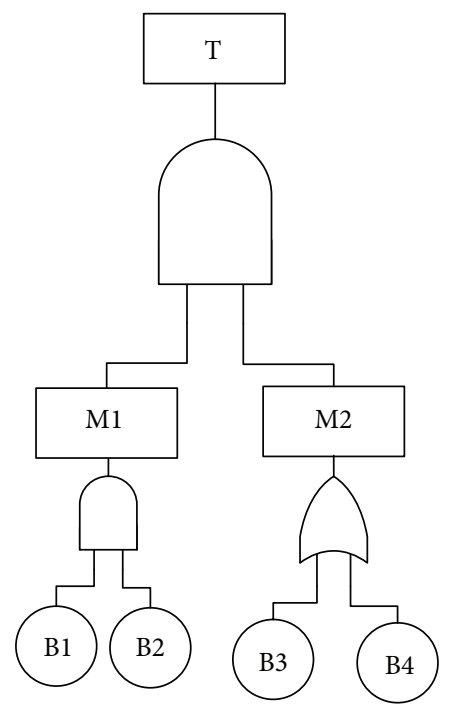

FIGURE 1: FT model for reliability analysis of a mechanical system.

$m_{i}: P \rightarrow N$ and is a marking whose $i$ th component represents the number of tokens in the $i$ th place. An initial marking is denoted by $m_{0}$. The tokens are pictured by dots.

A simple PN and its elements are shown in Figure 2. The four-tuple $(P, T, I, O)$ is called a $\mathrm{PN}$ structure that defines a directed graph structure. A PN models system dynamics using tokens and their firing rules.

Assume that every transition in a PN is associated with an exponentially distributed random delay from the enabling to the firing of the transition; then this $\mathrm{PN}$ is transformed into the stochastic Petri net (SPN), while every transition in a PN is associated with arbitrary distribution random delay; this $\mathrm{PN}$ is called ESPN. It is defined as follows [31].

An ESPN is a six-tuple $(P, T, I, O, m, H)$, where

$P=\left\{p_{1}, p_{2}, \ldots, p_{n}\right\}, n>0$, and is a finite set of places;

$T=\left\{t_{1}, t_{2}, \ldots, t_{s}\right\}, s>0$, and is a finite set of transitions, with $P \cup T=\Phi, P \cap T=\Phi$;

$I: P \times T \rightarrow N$ and is an input function that defines the set of directed $\operatorname{arcs}$ from $P$ to $T$, where $N=\{0,1,2 \ldots\}$;

$O: T \times P \rightarrow N$ and is an output function that defines the set of directed arcs from $T$ to $P$;

$m_{i}: P \rightarrow N$ and is a marking whose $i$ th component represents the number of tokens in the $i$ th place. An initial marking is denoted by $m_{0}$.

$H: T \rightarrow R$ is a vector whose component is a firing time delay with an extended/arbitrary distribution function.

Note that the firing time delay is the life time of corresponding component in ESPN model for reliability analysis.

2.2.2. Elements Transformation Rules of FT to ESPN. To obtain the ESPN, we introduce the transformation rules of

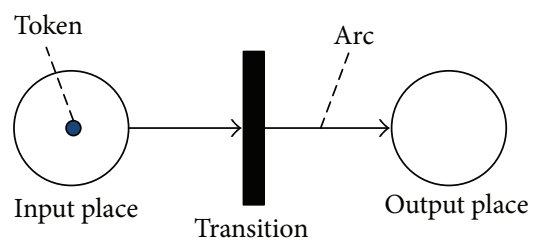

FIgURE 2: A simple PN and its elements.

elements of FT to ones of ESPN. Based on different logic relations, the transformation of $\mathrm{AND} / \mathrm{OR}$ gates of $\mathrm{FT}$ to AND/OR transitions of ESPN is shown in Figure 3.

As shown in Figure 3, the top/middle/basic event, logical gate, and logical relation line of FT are transformed to place, transition, and arc of ESPN, respectively.

2.2.3. The Establishment of ESPN Based on FT. Based on the FT and elements transformation rules of FT to ESPN, the FT based ESPN model for reliability analysis is obtained, as shown in Figure 4.

From Figures 4 and 1, compared to FT model, we can see that the building FT model needs 6 types of elements, that is, 3 types of events, 2 types of logic gates, and a kind of relation line, while the building ESPN model only needs 3 types of elements, that is, place, transition, and arc. Namely, the ESPN model is more concise than FT one. In addition, when each transition is associated with its corresponding life distribution function, it can achieve the real-time description of reliability analysis. Also, this model can achieve the dynamic delivery and propagation of reliability/fault information due to the introduction of transition and directed arc. Overall, the results denote that using ESPN method to establish the product reliability model is more convenient, concise, and effective than FT model.

\section{Method and Algorithm for Reliability Evaluation}

\subsection{Reliability Evaluation Method}

3.1.1. Reliability Evaluation Parameters and Calculation Meth$o d$. In this paper, the following two evaluation parameters of a system are adapted, that is, reliability degree of $R(x)$ and average life $E(x)$.

Let random variable $X$ denote the life for a specified system; then the unreliability $F(x)$ of the system at time $x$ is the probability of random variable $X \leq x$; namely,

$$
F(x)=\operatorname{Pr}(X \leq x) .
$$

The reliability of the system $R(x)$ is

$$
R(x)=1-F(x)=\operatorname{Pr}(X \geq x) .
$$

Let probability density function of the system be $f_{s}(x)$; then the average life $E(x)$ is

$$
E(x)=\int_{-\infty}^{+\infty} x f_{s}(x) d u .
$$




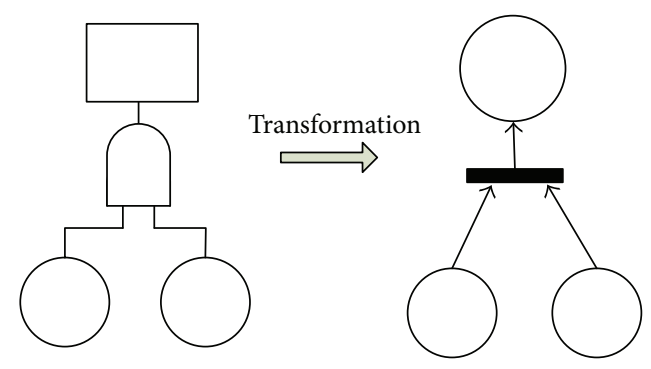

(a) Transformation of AND gate

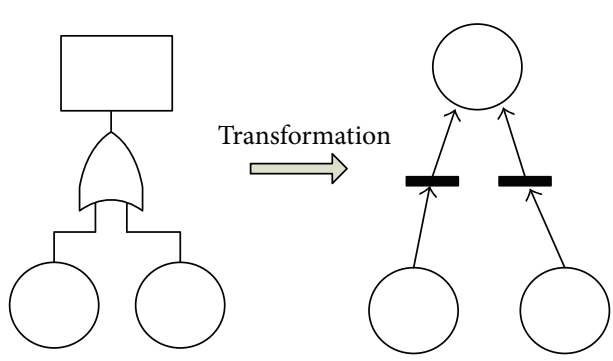

(b) Transformation of OR gate

FIGURE 3: Transformation of AND/OR gate.

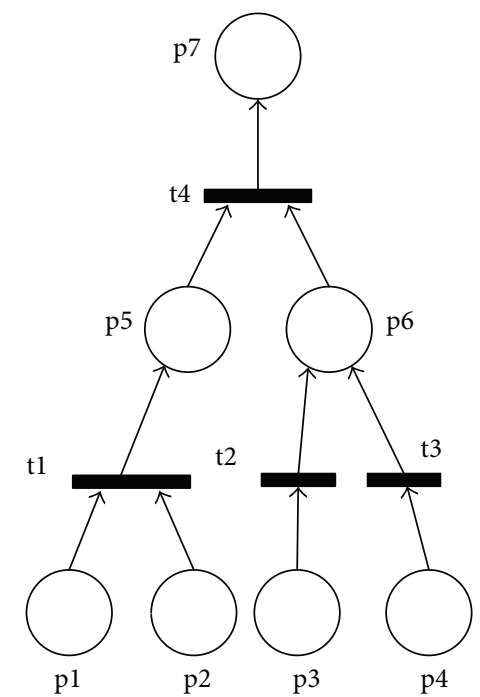

FIGURE 4: ESPN model for reliability analysis of a mechanical system.

In addition, for computer system reliability, the life calculation method of AND/OR transition in ESPN is pretended next.

Let a specified system consist of $n$ components and let the life of these $n$ components be $x_{1}, x_{2}, \ldots$, and $x_{n}$, respectively.

For AND transitions, the system life $x$ is expressed as

$$
x=\min \left\{x_{1}, x_{2}, \ldots, x_{n}\right\} .
$$

For OR transitions, the system life $x$ is expressed as

$$
x=\max \left\{x_{1}, x_{2}, \ldots, x_{n}\right\} .
$$

For example, in Figure 4, let the life of corresponding components of places $p_{1}$ and $p_{2}$ be $x_{1}$ and $x_{2}$, respectively; then the life of corresponding system $x_{5}$ of places $p_{5}$ is $x_{5}=$ $\min \left(x_{1}, x_{2}\right)$. Let the life of corresponding components of places $p_{3}$ and $p_{4}$ be $x_{3}$ and $x_{4}$, respectively; then the life of corresponding system $x_{6}$ of places $p_{6}$ is $x_{6}=\max \left(x_{3}, x_{4}\right)$.

For other systems, its life calculation can be obtained by the integration of AND and OR transition calculation. Such as, a system as shown in Figure 4, its system life $x_{7}=$ $\min \left\{x_{5}, x_{6}\right\}=\min \left\{\min \left(x_{1}, x_{2}\right), \max \left(x_{3}, x_{4}\right)\right\}$.
3.2. Algorithm for Reliability Evaluation. Stochastic simulation is an effective means to assess and calculate stochastic and probabilistic functions. It has effectively solved many stochastic programming problems [32-35]. While neural networks (NN) have been successfully used to solve many complex industrial evaluation and optimization problems due to their strong nonlinear fitting ability [36-38], we propose to use a stochastic simulation algorithm based on NN to solve the reliability of the proposed FT based ESPN model.

\subsubsection{Stochastic Simulation of Reliability Function}

Step 1. Initialize associated fault probability (life) distribution functions of each transition in ESPN model, and set the number of simulation cycles $M$.

Step 2. Generate the life time $x_{1}, x_{2}, \ldots$, and $x_{k}$ from their life distribution functions $f_{r 1}(x), f_{r 2}(x), \ldots$, and $f_{r k}(x)$.

Step 3. Based on transition transmission rules, that is, (4) and (5), a system life value $x$ is obtained from bottom to top in ESPN model.

Step 4. Repeat Steps 1-3 for $M$ times; namely, $M$ samples of system life are obtained.

Step 5. Calculation of the average value of $M$ samples of system life obtained in Step 4; that is, average life $E(x)$ of the system is obtained.

Step 6. Given a time $x^{\prime}$, record the number of $x>x^{\prime}$ in $M$ samples as $M^{\prime}$; then the system reality degree $R(x)$ is obtained; that is, $R(x)=M^{\prime} / M$.

3.2.2. Neural Networks (NN). NN is treated as a nonlinear mapping system consisting of neurons (processing units), which are linked by weighted connections. It usually consists of three layers: input, hidden, and output layers. There is an activation function in the hidden layer. It is defined as the sigmoid function in this paper [36-39].

Firstly, the method to determine the number of neurons of the input, hidden, and output layers is presented as follows.

The number of input neurons of an NN structure is the number of bottom places in ESPN model, namely, the number of basic events in FT model; in other words, the number of input neurons is the number of life distributions of basic fault component of a system in this paper. 
The number of output neurons is 1 representing one system life function. In terms of the $\mathrm{NN}$ structure, the main problem is to determine the best number of hidden neurons. The number can be infinite in theory, but finite in practice due to two reasons. Too many hidden neurons increase the training time and response time of the trained NN. On the other hand, too few hidden neurons make the NN lack of generalization ability. Therefore, it can usually be determined by the following formula; namely, $s=\sqrt{u+v}+b$, where $u$ and $v$ are the number of input neurons and output neurons, respectively, and $b$ is a constant from 1 to 10 [40]. Based on it, in terms of a system shown in Figure $4, u$ is set to be 4 since this system is composed of 4 bottom places, $v=1$; thus $s$ is a constant from 3 to 12 .

Secondly, backpropagation is the most commonly used method to calculate values for the weight and bias terms of an $\mathrm{NN}$ model. In this method, all weights are adjusted according to the calculated error term using a gradient method. Learning in an $\mathrm{NN}$, that is, the calculation of the weights of the connections, is achieved by minimizing the error between its output and the actual output over a number of available training data points. In this paper, the error term is controlled by the following MATLAB function, namely, net.trainParam.goal. It denotes the mean squared error between the output of the neural network and the actual output over a number of available training data points.

Thus the NN algorithm is presented as follows.

Step 1. Initialize the number of neurons at the input, hidden, and output layers, and initialize weight vector $w$.

Step 2. Calculate the output of the hidden layer and the output of output layer, and adjust the corresponding weights $w$.

Step 3. Calculate the error term, namely, training performance goal. If it is larger than the given error, go to Step 2, and otherwise, end.

3.2.3. NN Based Simulation Algorithm. Based on the presented stochastic simulation and $\mathrm{NN}$, the steps of the stochastic evaluation algorithm based on $\mathrm{NN}$ are presented as follows.

Step 1. Initialize the parameters of an NN structure and the number of training data points $K$.

Step 2. Establish an FT based ESPN model for reliability analysis based on fault logic relationships of each component in a system.

Step 3. Based on the relationship among the system life and the corresponding each component life in FT based ESPN model, generate the input-output data for $\mathrm{NN}$ training by the stochastic simulation technology.

Step 4. Train the NN to approximate the uncertain function, namely, the transition transmission rule/relation of the system life calculation, and obtain output data of a system life of the NN.

Step 5 . Forecast outputs of system life value are obtained by the NN algorithm.

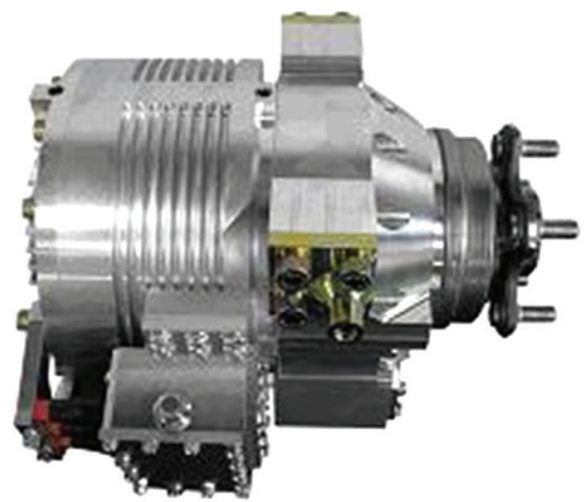

FIgURE 5: Motor of electric vehicle.

Step 6. Calculate reliability degree of $R(x)$ and average life $E(x)$ by stochastic simulation and obtained forecast outputs of system life value.

The above algorithm has been implemented in the MATLAB (R2009b) programming language.

\section{Reliability Modeling and Evaluation of Electric Vehicle Motor}

4.1. Reliability Modeling of Electric Vehicle Motor. As shown in Figure 5, it is a schematic graph of electric vehicle motor. It mainly consists of 3 parts, that is, stator, rotor, and axis. According to its components and their logical relation of fault occurrence, combined with the presented concept of FT, its FT model is shown in Figure 6.

Based on elements transformation rules of FA to ESPN, the FT based ESPN model of motor of electric vehicle is obtained and shown in Figure 7.

In addition, the paraphrase for the transitions and places of FT based ESPN model of electric vehicle motor is listed in Tables 1 and 2, respectively. Additionally, the life distribution types and parameters associated with each transition is listed in Table 2. Note that $\operatorname{Norm}(\mu, \sigma)$ and $\operatorname{Exp}(\lambda)$ denote normal and exponential distributions, respectively. The distribution parameters can be determined by the life test of motor and the unit of life time is hour [41].

4.2. Reliability Evaluation of Electric Vehicle Motor. The parameters of the NN based simulation algorithm are set as follows: for FT based ESPN model of electric vehicle motor, since there are 7 bottom places in this model, the number of input neurons is set to be $u=7$. Thus the number of hidden neurons is 12 by letting $b=12$. The given error term value is 0.000000004 . Based on the stochastic simulation, 5000 input-output data points are generated and obtained. Moreover, they are separated into two groups: 3000 data points for training and 2000 points for testing. Note that the number of training data points is set to be 5000 due to two reasons. First, too few data points make the solution of models inaccurate. Second, too many points increase the training time and response time of the solution model. Note 


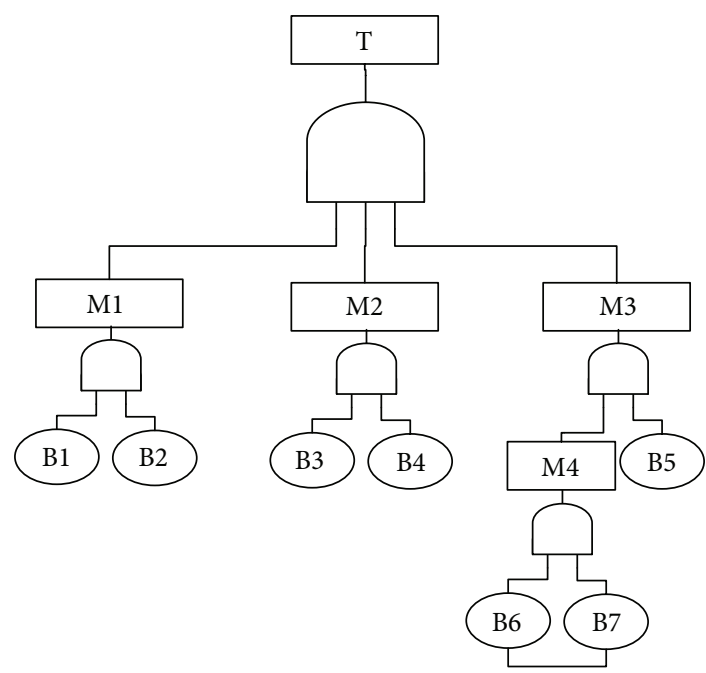

FIGURE 6: FT model of motor of electric vehicle.

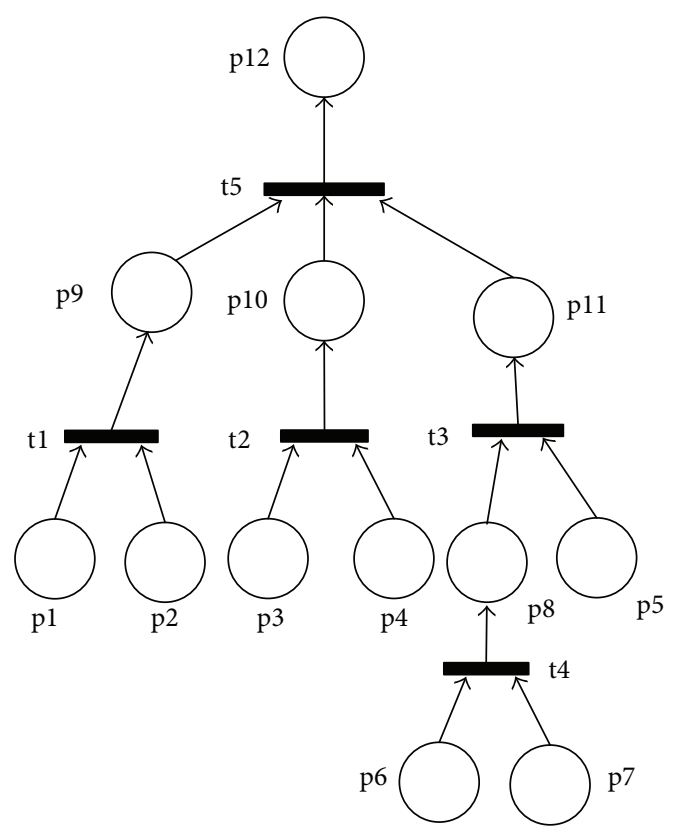

FIGURE 7: ESPN model of electric vehicle motor.

that the solution accuracy cannot be increased significantly with the number of data points $[36,37]$.

\subsubsection{The Results of Average Life E(x) of Electric Vehicle Motor.} Based on the established FT based ESPN model and the above presented algorithm, after the proposed stochastic simulation algorithms based on NN are executed, the average life $E(x)$ of electric vehicle motor is obtained; that is, $E(x)=4492.4$ hours. It denotes that the average use time of this electric vehicle motor is 4492.4 hours.

4.2.2. The Results of Reliability Degree $R(x)$ of Electric Vehicle Motor. Given the different running time $x$ of the electric vehicle motor, after the proposed stochastic simulation
TABLE 1: Events and paraphrases of each place in FT and ESPN models.

\begin{tabular}{lcc}
\hline FT & ESPN & Paraphrase \\
\hline B1 & p1 & Failure of core of the stator \\
B2 & p2 & Failure of winding of the stator \\
B3 & p3 & Failure of core of the rotor \\
B4 & p4 & Failure of winding of the rotor \\
B5 & p5 & Failure of bearing \\
B6 & p6 & Failure of the shaft \\
B7 & p7 & Failure of the bond \\
M4 & p8 & Failure of the shaft assembly \\
M1 & p9 & Failure of the stator \\
M2 & p10 & Failure of the rotor \\
M3 & p11 & Failure of the axis system \\
T & p12 & Failure of the motor system \\
\hline
\end{tabular}

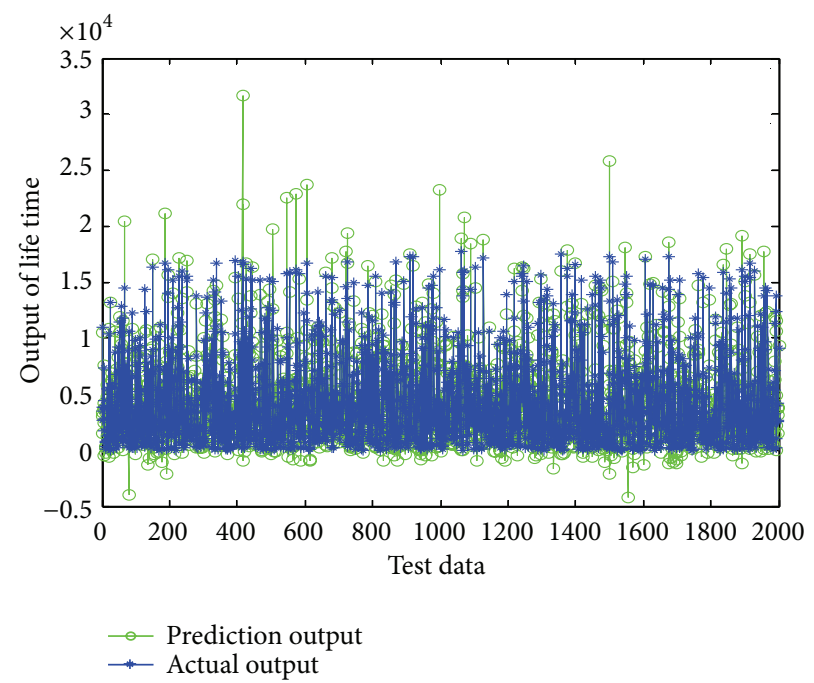

FIGURE 8: Prediction output of the proposed algorithm.

algorithms based on NN are executed, the corresponding reliability degree $R(x)$ is obtained, as listed in Table 3 .

As seen in Table 3, for example, $R(800)=0.871$; it denotes that the probability of this motor, which does not occur faulty, is 0.871 when it has run for 800 -hour time.

In addition, in order to make future tests and observe the effectiveness of the proposed methods, the predicted outputs of NN method and their output error between forecast and actual outputs at test data points are shown in Figures 8 and 9.

From Figures 8 and 9, the predicted and output results of the proposed algorithm are highly close. It reveals that the proposed algorithm can accurately achieve reliability evaluation of the electric vehicle motor.

\section{Conclusion}

Electric vehicle motor is one of key components in electric vehicle and has a great impact on the vehicle safety; thus it is important to perform its reliability analysis. To do 
TABLE 2: Paraphrases of each transition in ESPN models.

\begin{tabular}{|c|c|c|}
\hline ESPN & Paraphrase & Life distribution type $f(x)$ associated with transition $t$ \\
\hline$t_{1}$ & $\begin{array}{l}\text { Faults of core and winding of the stator cause fault of } \\
\text { stator }\end{array}$ & $\begin{array}{l}\text { Life distribution of core of the stator } x_{1} \text { is } \operatorname{Norm}(20000,1100) \text {. } \\
\text { Life distribution of winding of the stator } x_{2} \text { is } \operatorname{Exp}(16000)\end{array}$ \\
\hline$t_{2}$ & $\begin{array}{l}\text { Faults of core and winding of the rotor cause fault of the } \\
\text { rotor }\end{array}$ & $\begin{array}{l}\text { Life distribution of core of the rotor } x_{3} \text { is } \operatorname{Exp}(16000) \text {. } \\
\text { Life distribution of winding of the rotor } x_{4} \text { is } \operatorname{Norm}(18000,1000)\end{array}$ \\
\hline$t_{3}$ & $\begin{array}{l}\text { Faults of bearing and shaft assembly cause fault of the } \\
\text { axis system }\end{array}$ & $\begin{array}{l}\text { Life distribution of bearing } x_{5} \text { is } \operatorname{Exp}(12000) \text {. } \\
\text { Life distribution of shaft assembly is obtained by } x_{6} \text { and } x_{7}\end{array}$ \\
\hline$t_{4}$ & Faults of shaft and bond cause fault of shaft assembly & $\begin{array}{l}\text { Life distribution of shaft } x_{6} \text { is } \operatorname{Norm}(18000,1000) \text {. } \\
\text { Life distribution of bond } x_{7} \text { is } \operatorname{Norm}(16000,1100)\end{array}$ \\
\hline$t_{5}$ & $\begin{array}{l}\text { Faults of the stator, rotor, and axis system cause the fault } \\
\text { of the motor system }\end{array}$ & $\begin{array}{l}\text { Life distributions of stator, rotor, and axis system are obtained } \\
\text { by calculation of life of constituting its components }\end{array}$ \\
\hline
\end{tabular}

TABLE 3: Reliability degree of electric vehicle motor under different running time.

\begin{tabular}{lccccc}
\hline$x$ & 600 & 800 & 1000 & 1200 & 1400 \\
\hline$R(x)$ & 0.889 & 0.871 & 0.841 & 0.8111 & 0.778 \\
\hline
\end{tabular}

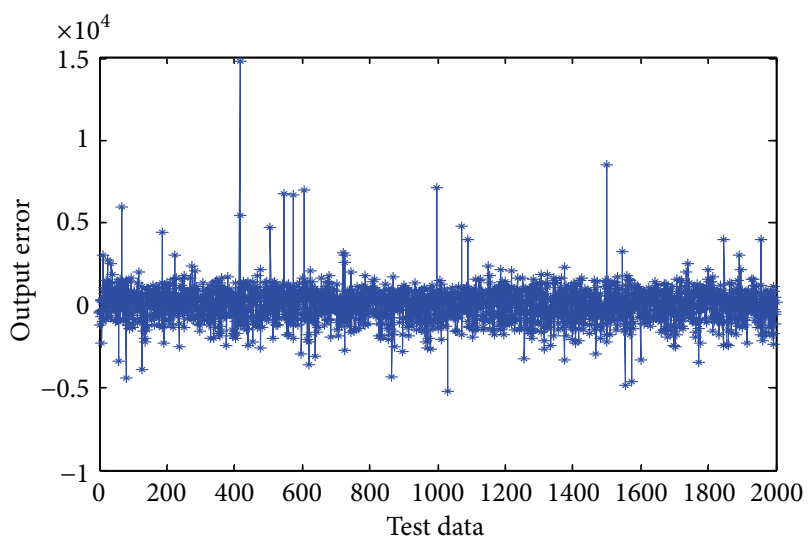

FIGURE 9: Output error of the proposed algorithm.

so, currently, researchers have discussed this problem by using FT analysis method. However, this method has many defects in analyzing the reliability of mechanical systems; for example, it cannot achieve the dynamic description of reliability and the building process of FT model needs a variety of elements. To deal with this problem, this paper proposes reliability modeling and evaluation issues of electric vehicle motor by using FT based extended stochastic Petri nets for the first time. Based on the concepts of FT and ESPN, combined with the defined transformation rules of their elements, an FT based ESPN model for reliability analysis of the mechanical system is obtained. In addition, the reliability calculation method is introduced on FT based ESPN model of the mechanical system and this work designs hybrid intelligent algorithms integrating stochastic simulation and $\mathrm{NN}$, namely, $\mathrm{NN}$ based simulation algorithm, to solve it. Finally, taking an electric vehicle motor as an example, its reliability modeling and evaluation issues are analyzed. The results reveal that they are feasible when used to solve the proposed problems. The obtained results can be used to guide decision makers in making better design when electric vehicle motor is developed and designed.

The future work is to find and use actual reliability test data to validate this method to provide the best decision support for reliability analysis of electric vehicle motor. This work merely analyzes the reliability of mechanical system of the electric vehicle motor. Thus, the reliability issue of integrated system integrating mechanical system and other software needs to be further discussed. In addition, advanced control and technology of electric vehicle motor should be further studied to improve its safety [42-44].

\section{Conflict of Interests}

The authors declare that there is no conflict of interests regarding the publication of this paper.

\section{Acknowledgments}

This work is financially supported by the Fundamental Research Funds for the Central Universities of China (DL13BB12), and China Postdoctoral Science Foundation Project under Grant 2013M541329.

\section{References}

[1] J. C. González Palencia, T. Furubayashi, and T. Nakata, "Energy use and $\mathrm{CO}_{2}$ emissions reduction potential in passenger car fleet using zero emission vehicles and lightweight materials," Energy, vol. 48, no. 1, pp. 548-565, 2012.

[2] T. F. Go, D. A. Wahab, M. N. A. Rahman, R. Ramli, and C. H. Azhari, "Disassemblability of end-of-life vehicle: a critical review of evaluation methods," Journal of Cleaner Production, vol. 19, no. 13, pp. 1536-1546, 2011.

[3] G. Tian, Y. Liu, H. Ke, and J. Chu, "Energy evaluation method and its optimization models for process planning with stochastic characteristics: a case study in disassembly decisionmaking," Computers \& Industrial Engineering, vol. 63, no. 3, pp. 553-563, 2012.

[4] G. Tian, M. Zhou, J. Chu, and Y. Liu, "Probability evaluation models of product disassembly cost subject to random removal time and different removal labor cost," IEEE Transactions on Automation Science and Engineering, vol. 9, no. 2, pp. 288-295, 2012. 
[5] G. Tian, J. Chu, Y. Liu, H. Ke, X. Zhao, and G. Xu, "Expected energy analysis for industrial process planning problem with fuzzy time parameters," Computers \& Chemical Engineering, vol. 35, no. 12, pp. 2905-2912, 2011.

[6] M. Burgess, N. King, M. Harris, and E. Lewis, "Electric vehicle drivers'reported interactions with the public: driving stereotype change?" Transportation Research F, vol. 17, pp. 33-44, 2013.

[7] P. Liu and H. P. Liu, "Application of z-source inverter for permanent-magnet synchronous motor drive system for electric vehicles," Procedia Engineering, vol. 15, pp. 309-314, 2011.

[8] X. Peng, H. Zhe, G. Guifang, X. Gang, C. Binggang, and L. Zengliang, "Driving and control of torque for direct-wheeldriven electric vehicle with motors in serial," Expert Systems with Applications, vol. 38, no. 1, pp. 80-86, 2011.

[9] C. Quinn, D. Zimmerle, and T. H. Bradley, "The effect of communication architecture on the availability, reliability, and economics of plug-in hybrid electric vehicle-to-grid ancillary services," Journal of Power Sources, vol. 195, no. 5, pp. 1500-1509, 2010.

[10] X. H. Zhu, S. M. Cui, N. Shi, and Y. L. Min, "Grey prediction model of motor reliability of electric vehicle," Electric Machines and Control, vol. 16, no. 8, pp. 42-46, 2012.

[11] X. H. Zhu, S. M. Cui, N. Shi, and Y. L. Min, "Grey prediction model of electric vehicle motor based on particle swarm optimization," High Voltage Engineering, vol. 38, no. 6, pp. 13911396, 2012.

[12] K. Mokhtari, J. Ren, C. Roberts, and J. Wang, "Application of a generic bow-tie based risk analysis framework on risk management of sea ports and offshore terminals," Journal of Hazardous Materials, vol. 192, no. 2, pp. 465-475, 2011.

[13] S.-R. Cheng, B. Lin, B.-M. Hsu, and M.-H. Shu, "Fault-tree analysis for liquefied natural gas terminal emergency shutdown system," Expert Systems with Applications, vol. 36, no. 9, pp. 11918-11924, 2009.

[14] A. Mentes and I. H. Helvacioglu, "An application of fuzzy fault tree analysis for spread mooring systems," Ocean Engineering, vol. 38, no. 2-3, pp. 285-294, 2011.

[15] J. Wu, S. Yan, and L. Xie, "Reliability analysis method of a solar array by using fault tree analysis and fuzzy reasoning Petri net," Acta Astronautica, vol. 69, no. 11-12, pp. 960-968, 2011.

[16] M. C. Zhou and F. Dicesare, Petri Net Synthesis for Discrete Event Control of Manufacturing Systems, Kluwer Academic, 1993.

[17] M. C. Zhou, Modeling, Simulation, and Control of Flexible Manufacturing Systems: A Petri Net Approach, World Scientific, 1999.

[18] H. S. Hu, M. C. Zhou, Z. W. Li, and Y. Tang, "An optimization approach to improved Petri net controller design for automated manufacturing systems," IEEE Transactions on Automation Science and Engineering, vol. 10, no. 3, pp. 772-782, 2013.

[19] Z. Li and M. Zhou, "Elementary siphons of Petri nets and their application to deadlock prevention in flexible manufacturing systems," IEEE Transactions on Systems, Man, and Cybernetics A: Systems and Humans, vol. 34, no. 1, pp. 38-51, 2004.

[20] M. Gao, M. Zhou, and Y. Tang, "Intelligent decision making in disassembly process based on fuzzy reasoning Petri nets," IEEE Transactions on Systems, Man, and Cybernetics B: Cybernetics, vol. 34, no. 5, pp. 2029-2034, 2004.

[21] A. Mhalla, S. Collart Dutilleul, E. Craye, and M. Benrejeb, "Estimation of failure probability of milk manufacturing unit by fuzzy fault tree analysis," Journal of Intelligent \& Fuzzy Systems, vol. 26, no. 2, pp. 741-750, 2014.
[22] N. Xie and A. Li, "Modeling and analysis of reconfigurable manufacturing system by extended stochastic petri nets," Jixie Gongcheng Xuebao/Chinese Journal of Mechanical Engineering, vol. 42, no. 12, pp. 224-231, 2006.

[23] G. Q. Huang, B. Zhang, and C. Z. Wang, "Network attack model based on hierarchical expanded stochastic Petri net," Computer Engineering, vol. 37, no. 22, pp. 12-18, 2011.

[24] A. Adamyan and D. He, "System failure analysis through counters of Petri net models," Quality and Reliability Engineering International, vol. 20, no. 4, pp. 317-335, 2004.

[25] B. Bertsche, Reliability in Automotive and Mechanical Engineering, Springer, Berlin, Germany, 2008.

[26] D. X. Liu and S. V. Amari, "Fault tree analysis," in Handbook of Performability Engineering, K. B. Misra, Ed., Springer, London, UK, 2008.

[27] W. Li, Y. Yao, Z. Ding, and Q. Pu, "Efficient mobile authentication scheme preserving user privacy for large-scale wireless networks," Ad Hoc \& Sensor Wireless Networks, vol. 17, no. 3-4, pp. 313-339, 2013.

[28] Z. Ding, J. Liu, J. Wang, and F. Wang, "An executable service composition code automatic creation tool based on Petri net model," Computing and Informatics, vol. 32, no. 5, pp. 968-986, 2013.

[29] L. Li, Z. J. Sun, M. C. Zhou, and F. Qiao, "Adaptive dispatching rule for semiconductor wafer fabrication facility," IEEE Transactions on Science and Engineering, vol. 10, no. 2, pp. 354-364, 2013.

[30] M. Zhou, D. Guo, and F. Dicesare, "Integration of Petri nets and moment generating function approaches for system performance evaluation," Journal of Systems Integration, vol. 3, no. 1, pp. 43-62, 1993.

[31] D. L. Guo, F. DiCesare, and M. C. Zhou, "A moment generating function based approach for evaluating extended stochastic Petri nets," IEEE Transactions on Automatic Control, vol. 38, no. 2, pp. 321-327, 1993.

[32] B. Liu, Uncertainty Theory: An Introduction to Its Axiomatic Foundations, vol. 154, Springer, Berlin, Germany, 2004.

[33] G. D. Tian, M. C. Zhou, and J. W. Chu, "A chance constrained programming approach to determine the optimal disassembly sequence," IEEE Transactions on Automation Science and Engineering, vol. 10, no. 4, pp. 1004-1013, 2013.

[34] G. Tian, Y. Liu, H. Zhang, J. Chu, and G. Xu, "Chance programming models for time-energy trade-off problem of product disassembly process with multiple stochastic variables," Advanced Science Letters, vol. 4, no. 4-5, pp. 1851-1854, 2011.

[35] M. A. Naeem, D. J. Dias, R. Tibrewal, P. C. Chang, and M. K. Tiwari, "Production planning optimization for manufacturing and remanufacturing system in stochastic environment," Journal of Intelligent Manufacturing, vol. 24, no. 4, pp. 717-728, 2013.

[36] V. N. Gaitonde and S. R. Karnik, "Minimizing burr size in drilling using artificial neural network (ANN)-particle swarm optimization (PSO) approach," Journal of Intelligent Manufacturing, vol. 23, no. 5, pp. 1783-1793, 2012.

[37] G. D. Tian, T. G. Qiang, J. W. Chu, G. Xu, and W. Zhou, "Efficiency optimization for disassembly tools via using NNGA approach," Mathematical Problems in Engineering, vol. 2013, Article ID 173736, 8 pages, 2013.

[38] U. Zuperl and F. Cus, "Optimization of cutting conditions during cutting by using neural networks," Robotics and ComputerIntegrated Manufacturing, vol. 19, no. 1-2, pp. 189-199, 2003. 
[39] V. S. Sharma, S. Dhiman, R. Sehgal, and S. K. Sharma, "Estimation of cutting forces and surface roughness for hard turning using neural networks," Journal of Intelligent Manufacturing, vol. 19, no. 4, pp. 473-483, 2008.

[40] G. D. Tian, T. G. Qiang, and J. W. Chu, "Influence factor analysis and prediction models for component removal time in manufacturing," Proceedings of the Institution of Mechanical Engineers B: Journal of Engineering Manufacture, vol. 227, no. 10, pp. 1533-1540, 2013.

[41] Y. L. Min, Research on life prediction and reliability testing method of the electric vehicle drive motor [M.S. thesis], Harbin Institute of Technology, 2011.

[42] W. Sun, H. Gao Sr., and O. Kaynak, "Finite frequency $H_{\infty}$ control for vehicle active suspension systems," IEEE Transactions on Control Systems Technology, vol. 19, no. 2, pp. 416-422, 2011.

[43] W. H. Sun, Z. Zhao, and H. J. Gao, "Saturated adaptive robust Control for active suspension systems," IEEE Transactions on Industrial Electronics, vol. 60, no. 9, pp. 3889-3896, 2013.

[44] W. H. Sun, H. J. Gao, and O. Kaynak, "Adaptive backstepping control for active suspension systems with hard constraints," IEEE Transactions on Mechatronics, vol. 18, no. 3, pp. 1072-1079, 2013. 


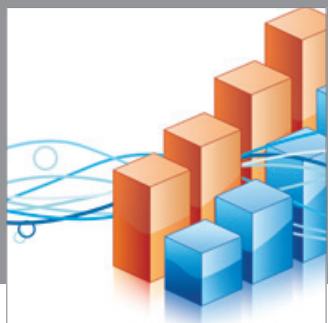

Advances in

Operations Research

mansans

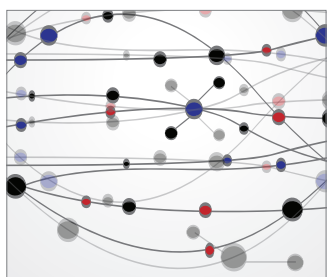

The Scientific World Journal
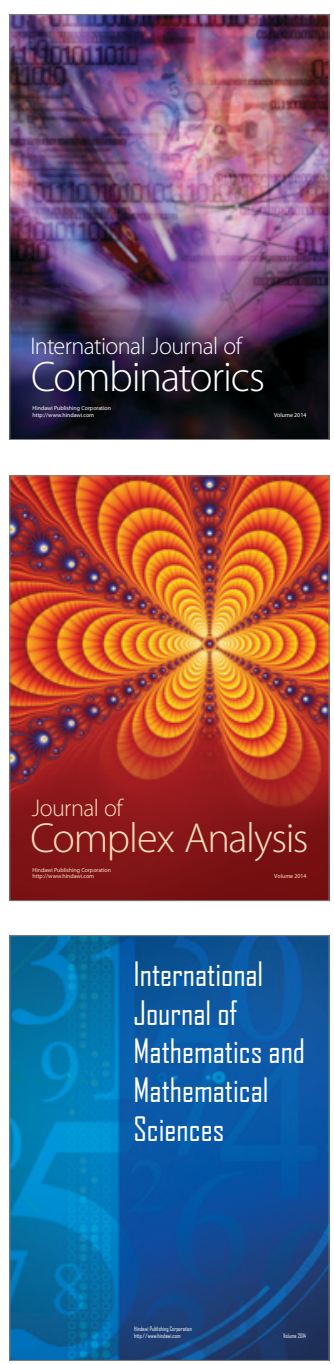
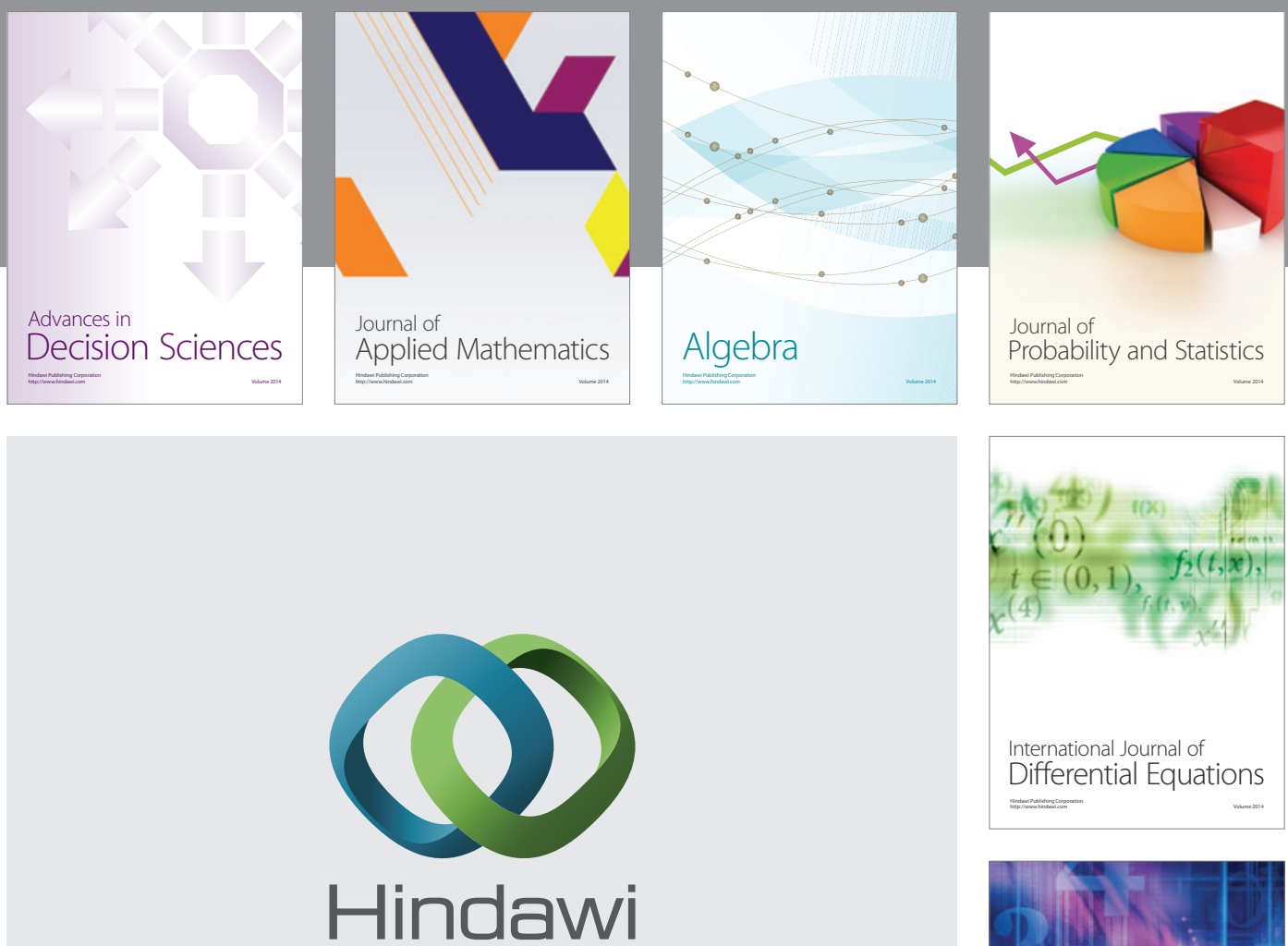

Submit your manuscripts at http://www.hindawi.com
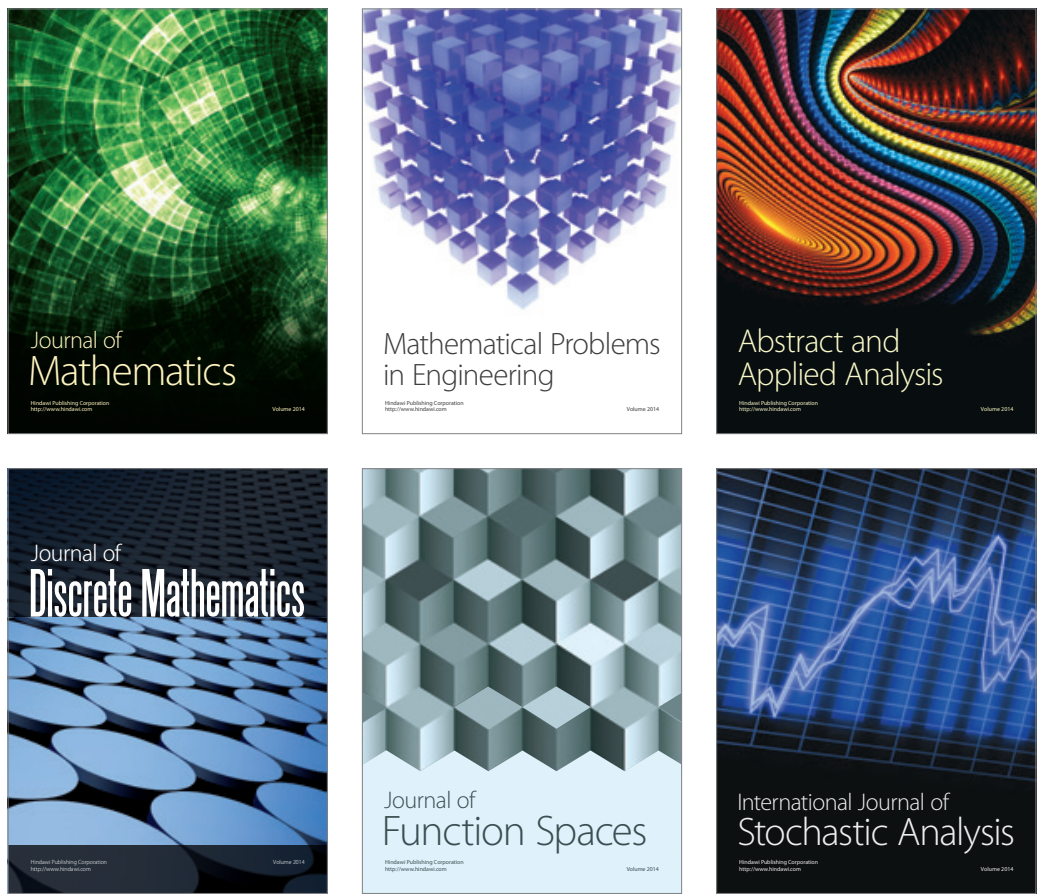

Journal of

Function Spaces

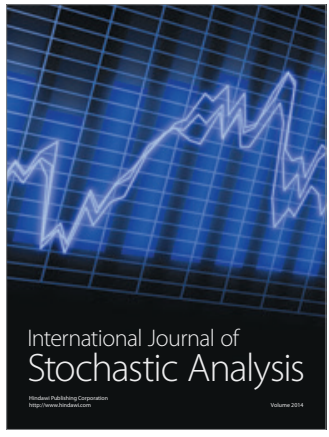

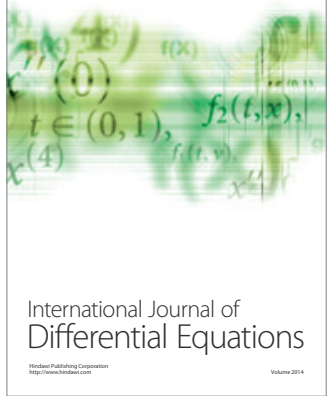
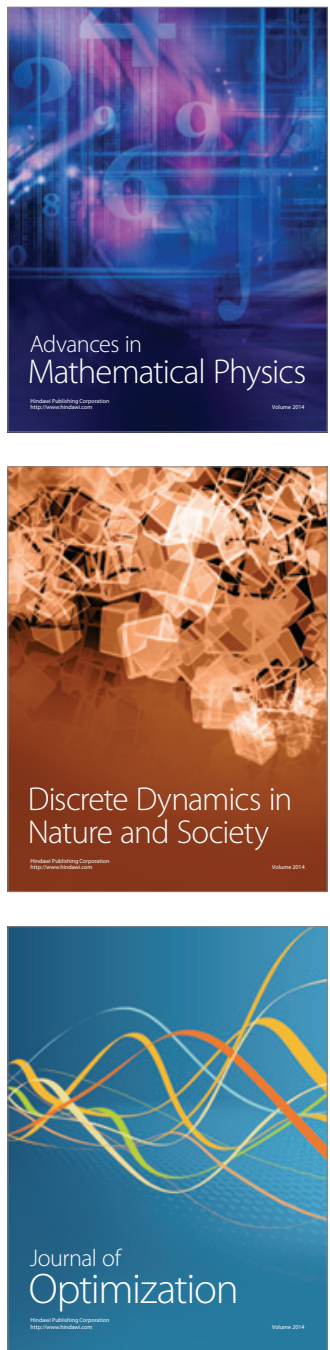